\title{
A hidden residential cell in the lung
}

\author{
Marc E. Rothenberg \\ Division of Allergy and Immunology, Cincinnati Children's Hospital Medical Center, Cincinnati, Ohio, USA.
}

\begin{abstract}
Eosinophils are classically known as proinflammatory cells, as they are equipped with a variety of preformed cytotoxic mediators and have been shown to definitively contribute to asthma. The connection between eosinophils and asthma development has led to a new class of asthma therapeutics based on blocking eosinophils with humanized antibodies that neutralize IL-5, a potent eosinophil growth, activation, and survival factor. Yet, recent studies have led to an increasing appreciation that eosinophils have a variety of homeostatic functions, including immunomodulation. In this issue of the $J C l$, Mesnil et al. identify a notable population of lungresident eosinophils and demonstrate that, compared with traditional eosinophils, these cells have distinct characteristics, including nuclear structure, surface markers, IL-5 independence, and immunoregulatory function that is capable of polarizing adaptive immune responses, at least in vitro. Thus, these results reinforce a key homeostatic role for this enigmatic cell population, particularly in residing and regulating immunity in the lung.
\end{abstract}

\section{Eosinophils: traditionally proinflammatory}

Eosinophils are traditionally viewed as proinflammatory cells, as they are equipped with a variety of preformed cytotoxic mediators, including the granule proteins major basic protein (MBP) and eosinophil peroxidase, which collectively account for more than half of an eosinophil's cellular mass (1). In addition, eosinophils are able to synthesize and release a variety of potent mediators, including leukotrienes, and pleiotropic cytokines, including TGF- $\beta$ (2). Yet, recent studies have shown that eosinophils also have a variety of homeostatic functions, including immunomodulation (3). For example, in the small intestine, which serves as the main reservoir for eosinophils at baseline, these cells have been shown to influence microbiotic content and mucus development and to be required for the production of secretory IgA, probably through production of $\operatorname{IL}-1 \beta(4,5)$. In addition, eosinophils have been identified at substantial levels in white and brown adipose tissue, and compelling data establish the existence of a key eosinophil/macrophage axis in weight gain and adipose tissue homeostasis (6). For example, under lean conditions, adipose tissue eosinophils are a main cellular source of IL-4, which polarizes macrophages into an alternatively activated state, which in turn improves the control of glucose metabolism by promoting secretion of insulin-sensitizing factors, such as catecholamines and IL-10 (ref. 7 and Figure 1).

\section{A resident cell population promotes immune homeostasis}

In this issue, Mesnil et al. (8) provide compelling evidence that extends the known roles of homeostatic eosinophils. In particular, the authors have identified a notable population of lung-resident eosinophils (referred to as rEos) and

\section{Related Article: p. 3279}

Conflict of interest: M.E. Rothenberg is a consultant for Receptos, PulmOne, and NKT Therapeutics; has an equity interest in Immune Pharmaceuticals, PulmOne, and NKT Therapeutics; receives royalties for reslizumab (Teva Pharmaceuticals); and is an inventor of patents owned by Cincinnati Children's Hospital Medical Center.

Reference information: J Clin Invest. 2016;126(9):3185-3187. doi:10.1172/JCI89768. demonstrate that these cells have characteristics that are distinct from those of traditional eosinophils, including an immunoregulatory function capable of polarizing adaptive immune responses, at least in vitro. The rEos express distinct levels of surface membrane markers, including the L-selectin receptor CD62L and the inhibitory receptor Siglec-F, and have a ringed nucleus (indicative of relative immaturity) compared with inflammatory eosinophils (referred to as iEoS), which express higher levels of Siglec-F and CD101 and lower levels of CD62L and mainly have donut-shaped nuclei. rEos exhibit ongoing piecemeal degranulation, suggesting that these cells are constantly releasing their contents. In contrast to iEos, which are mainly peribronchial, rEos reside in a distinct lung compartment, as they are located in the parenchyma. Unexpectedly, even though rEos express the IL- 5 receptor, this cell population is IL-5 independent and is not reduced in the presence of anti-IL-5. In contrast, iEos levels are attenuated under these same conditions. Consistent with unique regulation of $\mathrm{rEos}$, these $\mathrm{rEos}$ were not present at birth but achieved adult levels within 7 days after birth. The postnatal development of this cell population is in contrast to what has been reported for baseline gastrointestinal eosinophils, which are present at birth (2). Remarkably, in a house dust mite model of asthma, the levels and activation of rEos were not modified during induction of allergic airway inflammation, and rEos exhibited a distinct lack of change in gene expression levels, even when evaluated at the whole-genome level. Finally, using eosinophil-deficient mice ( $\triangle$ dblGATA), Mesnil and colleagues also presented evidence that rEos promote the development of Th1 immunity, either by directly inhibiting $\mathrm{T}$ cells or by impairing the ability of DCs to induce Th2 immunity (8). In vitro studies support the latter mechanism of rEos-mediated development of Th1 immunity. Table 1 summarizes the comparison between rEos and iEos. 


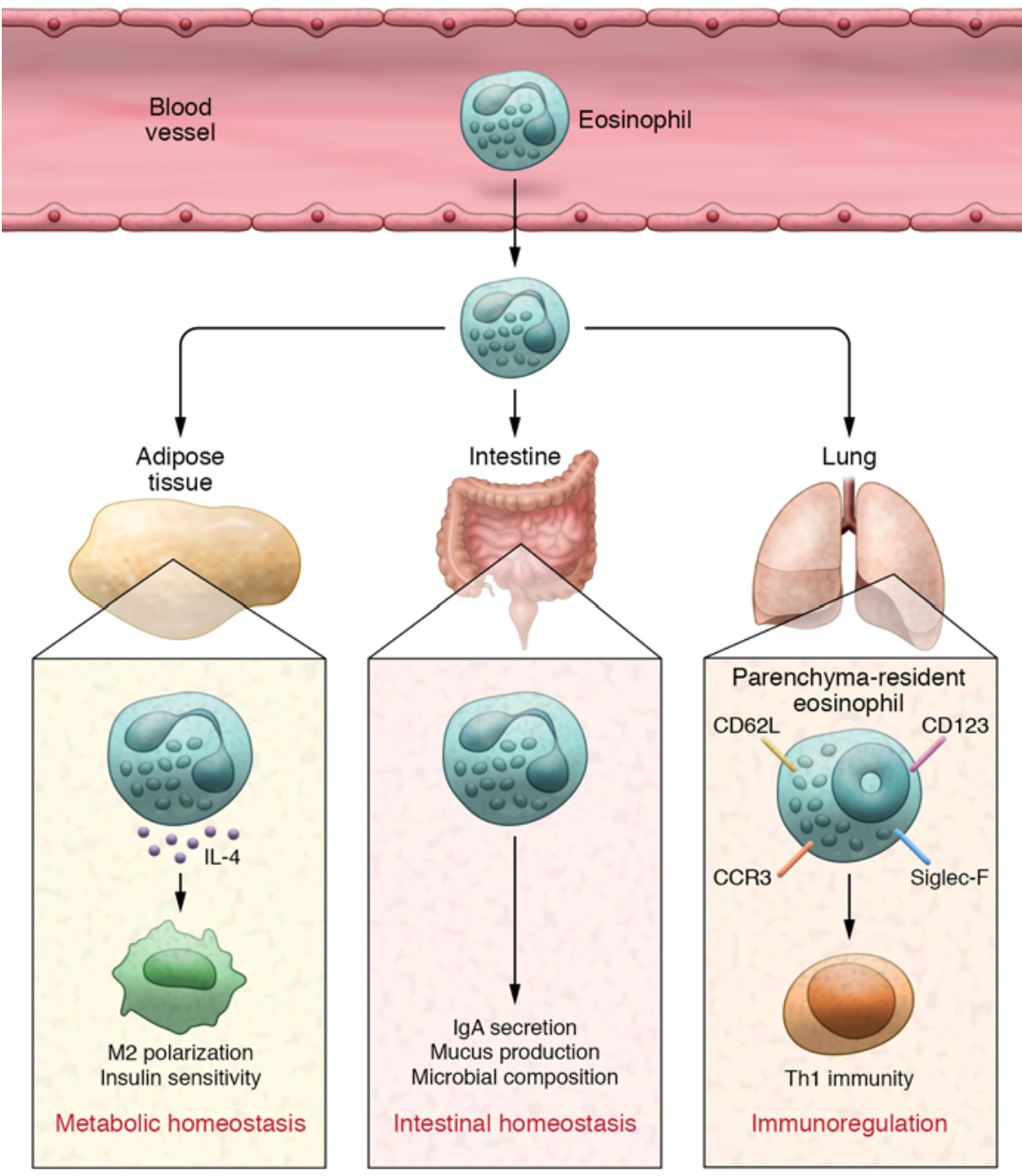

\section{Conclusions and future directions}

These are fascinating results, and Mesnil and colleagues (8) are to be commended for characterizing a difficult-to-study subpopulation of previously unappreciated eosinophils. At the same time, it is important to point out that these compelling findings are primarily limited to mice. While some preliminary evidence, primarily based on histologic analysis of lung samples, suggests that resident eosinophils may exist in the human lung, the two putative eosinophil populations in humans did not express the same differential molecular markers. Specifically, unlike in mice, the levels of CD101 or SIGLEC-8 (the human equivalent of murine Siglec-F) were comparable between iEos and rEos, whereas the levels of CD62L were variably different. Though Mesnil et al. present evidence that the IL-3 receptor differentiates the two eosin- ophil populations, the experiments compare tissue eosinophils with iEos present in induced sputum, populations that are probably different from each other for a number of reasons, including technical differences in how they were isolated. Even though the findings are limited to mice for the time being, they are notably

\section{Table 1. Differential properties of rEos and iEos}

Figure 1. Schematic diagram of the homeostatic roles of eosinophils. Eosinophils transit through the blood stream and home into various tissues at baseline. This schematic focuses on three tissues - adipose, small intestine, and lung. In adipose tissue, eosinophils regulate glucose levels and metabolism via eosinophil-derived IL-4, which regulates macrophage polarization and subsequent generation of insulin-sensitizing agents. In the small intestine, eosinophils regulate secretory IgA, mucus production and microbiota composition. A study in this issue by Mesnil et al. (8) show that in the lung, there is a substantial population of rEos in the parenchyma. These eosinophils have a ringed nucleus and express differential levels of Siglec-F and CD62L compared with inflammatory eosinophils and also express cardinal eosinophil markers including CCR3 and CD123 (the IL-5 receptor). Lung rEos have putative immunosuppressive function. Please note that the data in this figure are mainly derived from murine studies. important in identifying a new population of homeostatic lung eosinophils in this species (Table 1) and will undoubtedly be useful to the research community.

The results reported by Mesnil and colleagues (8) are somewhat surprising in regard to the putative Th2-suppressive effects of rEos. Eosinophils have been

$\begin{array}{lcc}\text { Property } & \text { rEos } & \text { iEos } \\ \text { Location } & \text { Lung parenchyma } & \text { Peribronchial } \\ \text { Nucleus } & \text { Ringed } & \text { Segmented } \\ \text { Allergen induced } & \text { No } & \text { Yes } \\ \text { Siglec-F levels } & \text { Intermediate } & \text { High } \\ \text { CD62L levels } & \text { High } & \text { Low } \\ \text { CD101 levels } & \text { Low } & \text { High } \\ \text { IL-5 dependent } & \text { No } & \text { Yes } \\ \text { Role } & \text { Immunosuppressive } & \text { Proinflammatory }\end{array}$


clearly identified as inflammatory cells, as now evidenced by the remarkable effects of the recently approved humanized antiIL-5 therapy for alleviating eosinophilic asthma $(9,10)$. The results reported by Mesnil et al. (8) raise potential concerns about the consequences of therapeutically ablating eosinophils in humans, as there are now several anti-eosinophil cytotoxic drugs in clinical development, including humanized anti-IL-5 receptor antibodies and anti-Siglec- 8 agents $(9,11)$. Though these drugs may benefit patients suffering from asthma who probably have an imbalanced iEos/rEos axis, patients with other eosinophilic diseases may become predisposed to asthma if human rEos are a homeostasis-maintaining population and depleted by these therapies. These possibilities will undoubtedly continue to spark the interest of researchers and clinicians focused on eosinophilic disorders.
Overall, we can conclude that eosinophils take up residence in the lung and that it is important to understand what they do in this newly identified location.

\section{Acknowledgments}

The author wishes to thank Shawna Hottinger for editorial assistance.

Address correspondence to: Marc E. Rothenberg, Division of Allergy and Immunology, Cincinnati Children's Hospital Medical Center, 3333 Burnet Ave. ML7028, Cincinnati, Ohio 45229, USA. Phone: 513.802.0257; E-mail: Rothenberg@cchmc.org.

1. Travers J, Rothenberg ME. Eosinophils in mucosal immune responses. Mucosal Immunol. 2015;8(3):464-475.

2. Rothenberg ME, Hogan SP. The eosinophil. Annu Rev Immunol. 2006;24:147-174.

3. Jacobsen EA, Helmers RA, Lee JJ, Lee NA. The expanding role(s) of eosinophils in health and disease. Blood. 2012;120(19):3882-3890.

4. Jung Y, et al. IL-1 $\beta$ in eosinophil-mediated small intestinal homeostasis and IgA production. Mucosal Immunol. 2015;8(4):930-942.

5. Chu VT, et al. Eosinophils promote generation and maintenance of immunoglobulin-A-expressing plasma cells and contribute to gut immune homeostasis. Immunity. 2014;40(4):582-593.

6. Lackey DE, Olefsky JM. Regulation of metabolism by the innate immune system. Nat Rev Endocrinol. 2016;12(1):15-28.

7. Qiu Y, et al. Eosinophils and type 2 cytokine signaling in macrophages orchestrate development of functional beige fat. Cell. 2014;157(6):1292-1308.

8. Mesnil C, et al. Lung-resident eosinophils represent a distinct regulatory eosinophil subset. J Clin Invest.2016; 126(9):3279-3295.

9. Fulkerson PC, Rothenberg ME. Targeting eosinophils in allergy, inflammation and beyond. Nat Rev Drug Discov. 2013;12(2):117-129.

10. Rothenberg ME. Humanized anti-IL-5 antibody therapy. Cell. 2016;165(3):509.

11. Bochner BS. "Siglec"ting the allergic response for therapeutic targeting. Glycobiology. 2016;26(6):546-552. 